\title{
Chemical topography of efferent projections from the median preoptic nucleus to pontine monoaminergic cell groups in the rat
}

\author{
Andrea M. Zardetto-Smith, Alan Kim Johnson* \\ Departments of Psychology, Pharmacology and the Cardiovascular Center, University of lowa, Iowa City, IA 52242, USA
}

Received 15 May 1995; revised version received 27 July 1995; accepted 31 August 1995

\begin{abstract}
This study examined efferent output from the median preoptic nucleus (MnPO) to pontine noradrenergic and serotonergic cell groups using an anterograde tracing technique (Phaseolus vulgaris leucoagglutinin, PHA-L) combined with glucose oxidase immunocytochemistry to serotonin (5-HT) or to dopamine- $\beta$-hydroxylase (DBH). Injections of PHA-L into the ventral MnPO resulted in moderate axonal labeling within the region of the B7 and B8 serotonergic groups in the dorsal raphe. PHA-L labeled fibers and punctate processes were observed in close apposition to many of the 5-HT immunoreactive neurons in these regions. In contrast, sparse terminal labeling was found within the B5 group in the raphe pontis nucleus, and only trace fiber labeling observed in the B3 and B6 groups. Efferents from the MnPO also provided moderate innervation to the A6 and A7 noradrenergic groups. PHA-L labeled punctate processes were found most frequently in close apposition to DBH-immunoreactive neurons at mid- to caudal levels of the locus coeruleus. Some labeled axons were also present within the A7 and A5 groups. Additionally, a close apposition between labeled MnPO efferents and 5-HT fibers within the lateral parabrachial nucleus was observed. The results indicate the MnPO provides a topographic innervation of monoaminergic groups in the upper brainstem.
\end{abstract}

Keywords: Serotonergic; Noradrenergic; Median preoptic nucleus; Raphe; Locus coeruleus; Anterograde tracing

The median preoptic nucleus (MnPo) is a major cell group in the periventricular anteroventral third ventricular (AV3V) region and has been hypothesized to be a critical integrative area of peripherally derived hydration-related information. Activity of neural inputs into this area has been proposed to reflect plasma concentrations of fluid balance-associated hormones (e.g. angiotensin II via action on the subfornical organ) and firing frequency of systemic blood pressure/volume receptor afferents that project into the brain via the nucleus of the solitary tract (NTS) $[9,21]$. Given the role of the MnPO in processing interoceptive signals related to the maintenance of extracellular fluid volume, the efferent projections of this nucleus are crucial in determining the effector pathways through which this integration is expressed. Previous anatomical studies have demonstrated the $\mathrm{MnPO}$ is densely innervated by noradrenergic immunoreactive

\footnotetext{
* Corresponding author. Department of Psychology, University of Iowa, 1 I Seashore Hall E., Iowa City, IA 52242-1407, USA. Tel.: + I $3193352423 ;$ fax: + 3193350191 .
}

fibers [20,21] in part originating from the A2 group in the NTS [15]. In addition to this major noradrenergic input, the $\mathrm{MnPO}$ receives efferents from brainstem serotonergic groups (for review, see [18]). While the ascending projections from these monoaminergic groups to the $\mathrm{MnPO}$ have been documented, reciprocal descending projections from the MnPO to serotonergic neurons in the dorsal raphe and the noradrenergic neurons in the A6-A7 areas have not been described. Physiological studies have indicated noradrenergic innervation of the MnPO may function to enhance the action of angiotensin II in the ventral portion of the AV3V region [9], which in turn may influence the output of MnPO neurons to hypothalamic and brainstem autonomic areas that also participate in the maintenance of extracellular fluid volume [21]. Less is known about the functional significance of the serotonergic innervation of the $\mathrm{MnPO}$, but recent studies have implicated central serotonergic mechanisms in the control of sodium excretion and water intake $[10,11,17]$, possibly modulated (in part) by efferent projections originating from the MnPO. The purpose of the present study was to 


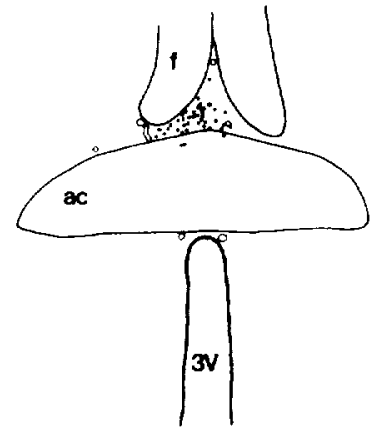

MP 3

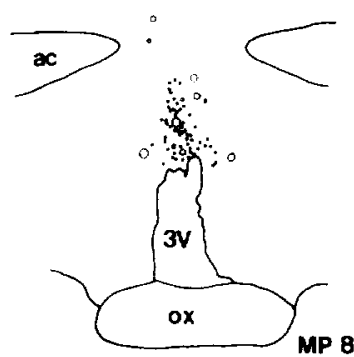

Fig. I. Line drawing illustrating representative injection sites of PHA-L into the MnPO. (A) Coronal section illustrating an injection into the dorsal MnPO (case MP 3) at a level approximately $-0.30 \mathrm{~mm}$ posterior to bregma (as per Paxinos and Watson. [13]). (B) Injection into the ventral MnPO (case MP 18) at a level approximately $-0.26 \mathrm{~mm}$ posterior to bregma (as per Paxinos and Watson, [13]). Closed circles represent labeled cell bodies. Maximal PHA-L fiber and terminal labeling in MnPO efferent target nuclei occurred in cases such as MP 18, where the injection was centered within the ventral MnPO. ac, anterior commissure; ox, optic chiasm; $3 \mathrm{~V}$, third ventricle.

examine the innervation of noradrenergic/serotonergic cell groups in the upper brainstem by efferents from the $\mathrm{MnPO}$ using a combination of anterograde tracing with Phaseolus vulgaris leucoagglutinin (PHA-L) and glucose oxidase immunocy tochemistry.

Male Long-Evans rats $(n=6)$ were anesthetized with sodium pentobarbital $(55 \mathrm{mg} / \mathrm{kg}$ ) and placed in a stereotaxic apparatus. Iontophoretic injections of PHA-L (Vector) were delivered into the median preoptic nucleus (MnPO) according to stereotaxic coordinates derived from the Paxinos and Watson atlas [13] via glass micropipettes (tip diameter $10-15 \mu \mathrm{m}$ ). Cathodal current $(5 \mu \mathrm{A})$ was delivered in $7 \mathrm{~s}$ pulses every $14 \mathrm{~s}$ over a $30 \mathrm{~min}$ period (Midgard Electronics constant current device).

Ten to 14 days later, the animals received a lethal dose of sodium pentobarbital and were perfused using the $\mathrm{pH}$ variation method [2]. Coronal sections $(20 \mu \mathrm{m})$ of the brain were cut using a vibratome and immunocytochemically processed according to the method of Gerfen and Sawchenko [7]. Following processing for PHA-L labeling, the tissue was washed for 4-5 days in phosphate buffered saline. Alternate adjacent sections were processed for serotonin (5-HT) and dopamine- $\beta$-hydroxylase
(DBH) immunoreactivity according to the method of Piekut [14]. Rabbit anti-5-HT (Biogenex Labs) and rabbit anti-DBH (IncStar) were used at a 1:1000 dilution. Sections were rinsed in phosphate buffered saline, mounted on acid-cleaned gel-coated slides, and cover-slipped with Permount. Slides were photographed using a Nikon FX microscope equipped with Nomarski optics.

Maximal PHA-L labeling was observed in brains in which the injection was centered within the ventral portion of the MnPO (Fig. 1). In comparison, injections which were centered in the dorsal portion of the MnPO, that is, located above the anterior commissure $(n=2)$, resulted in lighter anterograde label in known terminal regions of the $\mathrm{MnPO}$ [21].

PHA-L immunoreactive axons labeled by a brown reaction product were visible as varicose processes with punctate terminations. Neurons immunoreactive to 5-HT or DBH were identified by a blue reaction product filling the cytoplasm and in certain areas, such as the locus coeruleus, by heavily labeled dendritic processes as well. The distribution of the 5-HT and DBH-immunoreactive neurons corresponded to previous descriptions of serotonergic and noradrenergic groups within the pons (for review, see $[4,8,18]$ ) and their same numerical terminology has been utilized in the following descriptions.

Beginning caudally, a few PHA-L labeled fibers could be traced to the B3 serotonergic group within the raphe magnus nucleus. Sparse PHA-L label was observed in the B5 serotonergic group in the raphe pontis nucleus and dorsally, medial to the medial longitudinal fasiculus. PHA-L labeled fibers in this area were typically fine in structure. A few fibers were present in the B6 group of cells, lying medially on the floor of the fourth ventricle. The densest PHA-L labeled processes within serotonergic groups within the pons was found in the rostrocaudal extent of the nucleus raphe dorsalis (Fig. 2A-D). Neurons located in the ventromedian portion of the nucleus raphe dorsalis (the $\mathrm{B} 8$ group) received a moderate innervation from the MnPO (Fig. 2B). In the B7 group, PHA-L labeled punctate processes were found in close apposition to 5-HT labeled neurons within its dorsomedian mesencephalic part. Several 5-HT labeled neurons located in the mesencephalic 'wing' of the group [3] also received innervation from the MnPO (Fig. 2C,D).

The A6 noradrenergic group, particularly DBH-posi-

Fig. 2. Photomicrographs illustrating MnPO efferent label in monoaminergic groups of the pons. (A) Low-power photomicrograph (13.8 $\times$ ) illustrating serotonergic neurons in the nucleus centralis superior (B8; indicated by the diamond-shaped box in the midline) and the nucleus raphe dorsalis (B7; upper box). Innervation of the $\mathbf{B 8}$ group by MnPO efferents is shown in higher power in (B), while innervation of the B7 group is shown in detail in (C,D). (B) High-power $(221 \times)$ photomicrograph utilizing Nomarski optics demonstrating the interaction of PHA-L labeled fibers and terminals (brown) with serotonergic-immunoreactive cell bodies (blue) in the ventromedian portion of the nucleus raphe dorsalis (B8 group). (C,D) Higher power photomicrographs ((C), 140X; (D), 221X) utilizing Nomarski optics showing innervation (arrows) of serotonergic-immunoreactive neurons (blue) in the lateral portion of the B7 group by MnPO efferents (brown). (E,F) Low (E; 68.5X) and high (F; 140X)-power photomicrographs utilizing Nomarski optics showing close apposition (arrows) of DBH-immunoreactive neurons (blue) in the lateral portion of the A6 group with MnPO efferents (brown). $(\mathrm{G}, \mathrm{H})$ Photomicrographs utilizing Nomarski optics showing close apposition (arrows) of 5-HT-immunoreactive fibers (blue) in the lateral parabrachial with MnPO efferents (brown). Magnification: (G), 140X; (H), 431 X. 


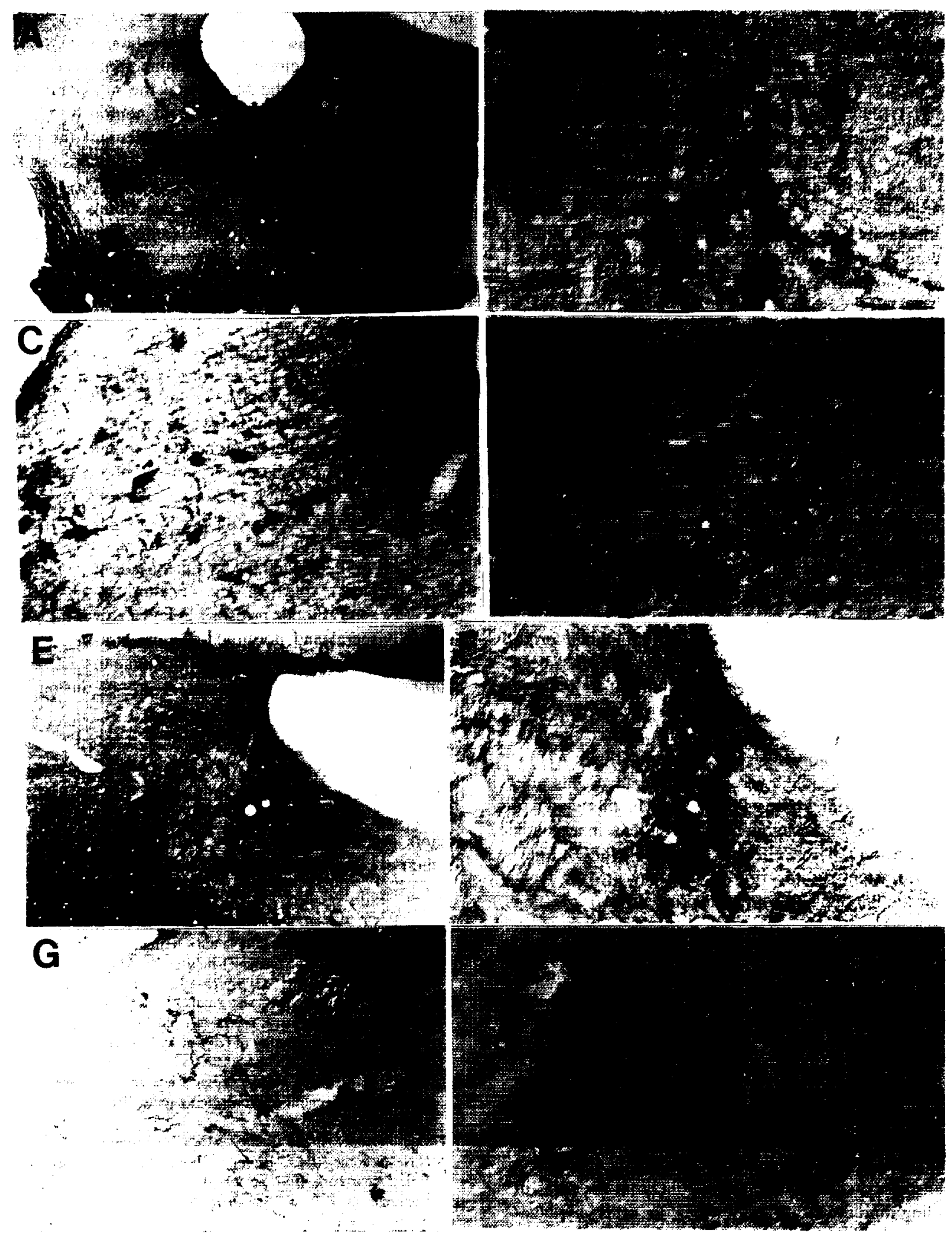


tive neurons located in the mid- to caudal parts of the locus coeruleus, also received moderate innervation from the MnPO (Fig. 2E,F). A number of PHA-L labeled fibers were observed in areas containing a high density of $\mathrm{DBH}$ immunolabeled dendrites surrounding the locus coeruleus. Only trace amounts of PHA-L labeled fibers were found in this area containing the $A 7$ group and appositions to DBH-immunoreactive neurons were less common. An occasional punctate process appeared in close apposition to DBH-stained neurons in the A5 group, located in the lateral and ventral reticular formation.

Within the pons at the level of the A6 group, 5-HTimmunoreactive fibers and terminals were noted within the lateral parabrachial nucleus (IPB) (Fig. 2G,H). PHA-L labeled efferents from the MnPO appeared to interact with these fibers, so that the PHA-L-labeled immunoreactive boutons were in extremely close apposition to 5HT-labeled fibers in the central lateral and dorsal lateral subnuclei (as per the terminology of Fulwiler and Saper [6]).

The results demonstrate that the MnPO innervates the upper brainstem serotonergic and noradrenergic groups topographically. The A6 noradrenergic group in the locus coeruleus was the most heavily innervated, with sparse innervation of the A5 and A7 noradrenergic groups. $\mathrm{MnPO}$ efferent processes frequently were closely apposed to cells of the B7 and B8 serotonergic groups of the dorsal raphe, but sparsely innervated the B6, B5 and B3 groups.

The integrity of the structures along the lamina terminalis, including the ventral portion of the $\mathrm{MnPO}$, has been shown to be critical for water and sodium intake induced by several experimental protocols (for review, see [9]). The physiological significance of 5-HT input to the lamina terminalis has not been fully determined, but it is interesting to note that the serotonergic input to this area arises mainly from groups [12] shown in this study to receive the heaviest amount of reciprocal innervation from the MnPO. Thus, efferents from the MnPO to particular brainstem serotonergic groups may provide feedback in modulating the forebrain projections originating from these areas.

Previous studies have indicated that an important interaction between angiotensin II and norepinephrine occurs in the ventral portion of the AV3V region, such that a minimal amount of norepinephrine must be present in this area for elicitation of normal drinking and pressor responses to exogenously administered angiotensin II [1]. The noradrenergic input from the lower brainstem to the $\mathrm{MnPO}$ has been documented to arise primarily from the A2 group in the NTS, with the A1 noradrenergic contribution to the AV3V region being small [15]. Although it has not been documented, it seems likely on the basis of ascending noradrenergic projections to other hypothalamic nuclei [5] that cells in the A6 group also contribute to the noradrenergic innervation of the MnPO. Assuming this speculation were true, it is therefore not surprising to find the major termination of $\mathrm{MnPO}$ efferents in upper brainstem noradrenergic groups in the A6, and not the A5 or A7, cell clusters.

Extracellular fluid volume homeostatic mechanisms acting through the circuitry of lamina terminalis structures which includes the MnPO and the monoaminergic system, may interact at a number of central autonomic sites, including the paraventricular nucleus of the hypothalamus (PVN) and the IPB. Serotonin-immunoreactive fibers innervate both the MnPO and the PVN $[12,16]$, though there are no data available describing the degree of collateralization (if any) of efferents from brainstem serotonergic nuclei to these two forebrain groups. Efferents from the MnPO terminate largely within the parvocellular portion of the PVN [21], where the terminals of serotonergic fibers arising from the raphe are concentrated [12]. Water deprivation increases vasopressin secretion by activating 5-HT neurons and experimental evidence suggests this may occur indirectly via an interneuron [19]. The MnPO also innervates the IPB [20] and recent work indicates that serotonergic mechanisms associated with the IPB may be involved in an inhibitory system functioning to limit fluid intake in conditions associated with hypovolemia [10]. Finally, serotonergic neurons may act centrally in both a sympathoexcitatory (via 5$\mathrm{HT}_{2}$ receptors), as well as sympathoinhibitory (via 5$\mathrm{HT}_{\mathrm{IA}}$ receptors), role [3]. The concomitant changes in heart rate and blood pressure relayed to the $\mathrm{MnPO}$ through visceral and hormonal feedback may influence efferent output from the MnPO to modulate the activity of neurons within these monoaminergic groups during central autonomic activation.

Supported by NRSA F32HL-08349, the Iowa Affiliate of the American Heart Association, the NIH (HLBI) HL14338 and HL-44546, and NASA NAGW-4358. The authors gratefully thank Terry Beltz and Paul Reimann for their excellent technical assistance.

[1] Bellin, S.I., Landas, S.K. and Johnson, A.K., Localized injections of 6-hydroxydopamine into lamina terminalis-associated structures: effects on experimentally induced drinking and pressor responses, Brain Res., 416 (1987) 75-83.

[2] Berod, A., Hartman, B.K. and Pujol, J.F., Importance of fixation in immunocytochemistry: use of formaldehyde solutions at variable $\mathrm{pH}$ for the localization of tyrosine hydroxylase, J. Histochem. Cytochem., 29 (1981) 844-850

[3] Chaouloff, F., Physiopharmacological interactions between stress hormones and central serotonergic systems, Brain Res. Rev., 18 (1993) $10-32$

[4] Consolazione, A. and Cuello, A.C., CNS serotonin pathways. In N.N. Osborne (Ed.), Biology of Serotonergic Transmission, Wiley, New York, 1982, pp. 29-61

[5] Cunningham, E.T and Sawchenko, P.E., Anatomical specificity of noradrenergic inputs to the paraventricular and supraoptic nuclei of the rat hypothalamus, J. Comp. Neurol., 274 (1988) 60-76.

[6] Fulwiler. C.E. and Saper. C.B., The subnuclear organization of 
the efferent connections of the parabrachial nucleus in the rat, Brain Res. Rev., 7 (1984) 229-259.

[7] Gerfen, C.R. and Sawchenko, P., An anterograde neuroanatomical tracing method that shows the detailed morphology of neurons, their axons, and terminals: immunohistochemical localization of an axonally transported plant lectin, Phaseolus vulgaris leuocoagglutinin (PHA-L), Brain Res, 290 (1984) 219-238.

[8] Hokfelt, T., Martensson, R., Bjorklund, A., Kleinau, S. and Goldstein, M., Distribution maps of tyrosine-hydroxylase-immunoreactive neurons in the rat brain. In $A$. Bjorklund and $T$. Hokfelt (Eds).. Handbook of Chemical Neuroanatomy, Vol. 2: Classical Transmitters in the CNS, Part I, Elsevier, Amsterdam, 1984, pp. 277-379.

[9] Johnson, A.K. and Edwards, G.L., The neuroendocrinology of thirst: afferent signaling and mechanisms of central integration, Curr. Top. Neuroendocrinol., 10 (1990) 149-190.

[10] Menani, J.V., and Johnson, A.K., Lateral parabrachial serotonergic mechanisms: angiotensin-induced pressor and drinking resposes, Am. J. Physiol., 269 (Regulatory Integrative Comp. Physiol. 38) (1995) in press.

[11] Montes, R. and Johnson, A.K., Efferent mechanisms mediating renal sodium and water excretion induced by centrally administered serotonin, Am. J. Physiol., 259 (1990) R I267-R1273

[12] Moore, R.Y., Halaris, A.E. and Jones. B.E., Serotonin neurons of the midbrain raphe: ascending projections, J. Comp. Neurol., 180 (1978) 417-438

[13] Paxinos, G. and Watson, C., The Rat Brain in Stereotaxic Coordinates, Academic Press, Orlando, 1986.

[14] Piekut, D.T., Interactions of immunostained ACTH ${ }^{1-39}$ fibers and
CRF neurons in the paraventricular nucleus of rat hypothalamus: application of avidin-glucose oxidase to dual immunostaining procedures, J. Histochem. Cytochem., 35 (1987) 261-265.

[15] Saper, C.B, Reis, D.J. and Joh, T., Medullary catecholamine inputs to the anteroventral third ventricular cardiovascular regulatory region in the rat, Neurosci. Lett., 42 (1983) 285-291.

[16] Sawchenko, P.E., Swanson, L.W., Steinbusch, H.W.M. and Verhofstad. A.A.J. The distribution and cells of origin of serotonergic inputs to the paraventricular and supraoptic nuclei of the rat, Brain Res., 277 (1983) 355-360.

[17] Stein, J.M., Lind, R.W. and Johnson, A.K., Central serotonergic influences on renal electrolyte and water secretion, Neuropharmacology, 26 (1987) 1685-1692.

[18] Steinbusch. H.W.M., Serotonin-immunoreactive neurons and their projections in the CNS, In A. Bjorklund and T. Hokfelt (Eds.), Handbook of Chemical Neuroanatomy, Vol. 3: Classical Transmitters and Transmitter Receptors in the CNS, Part II, Elsevier, New York, 1984, pp 68-125.

[19] Van de Kar, L.D., Neuroendocrine pharmacology of serotonergic (5-HT) neurons, Annu. Rev. Pharmacol. Toxicol., 31 (1991) 289320.

[20] Zardetto-Smith, A.M., Beltz, T.G. and Johnson, A.K., Chemoarchitecture of the rat median preoptic nucleus, Third IBRO World Congr. Neurosci., (1991) 130.

121] Zardetto-Smith, A.M., Thunhorst, R.L., Cicha, M.Z. and Johnson. A.K., Afferent signaling and forebrain mechanisms in the behavioral control of extracellular fluid volume, Ann. N. Y. Acad. Sci., 689 (1993) 161-176. 


\section{.}

